\title{
Dynamic response of artificial bipolar molecules
}

\author{
Egidijus Anisimovas* and F. M. Peeters ${ }^{\dagger}$ \\ Departement Natuurkunde, Universiteit Antwerpen (UIA), B-2610 Antwerpen, Belgium
}

(Received 27 March 2002; published 5 August 2002)

\begin{abstract}
We calculate the equilibrium properties and the dynamic response of two vertically coupled circular quantum dots populated by particles of different electrical charge sign, i.e., electrons and holes. The equilibrium density profiles are obtained and used to compute the frequencies and oscillator strengths of magnetoplasma excitations. We find a strong coupling between the modes derived from the center-of-mass modes of the individual dots which leads to an anticrossing with a pronounced oscillator strength transfer from the "acoustic" to the "optical" branch. Also, due to the breaking of the generalized Kohn theorem a number of other than center-of-mass modes are excited whose oscillator strengths, however, are rather weak.
\end{abstract}

DOI: 10.1103/PhysRevB.66.075311

PACS number(s): 73.21.La, 78.67.Hc

\section{INTRODUCTION}

The physics of quantum dots-the small man-made structures in a semiconductor containing anything from a few to thousands or more electrons - has already enjoyed a busy and fruitful decade as a major field of research in condensed matter physics. ${ }^{1-3}$ A large part of the experimental work on quantum dots focused on the probing of the electronic states inside the dots by means of far-infrared (FIR) spectroscopy. ${ }^{4-7}$ However, a very common feature of many quantum structures is their nearly circular shape and a parabolic confining potential ${ }^{8,9}$ that has profound consequences on the optical response. According to the generalized Kohn theorem, ${ }^{10}$ under such conditions the center-of-mass (c.m.) motion decouples from the relative motion of the electrons, and the electric field of the FIR radiation couples only to the former. Consequently, the absorption spectra of the circular parabolic quantum dots consist only of two c.m. peaks whose positions are independent of the electron number and insensitive to the electron-electron interaction effects.

Actual experiments performed on arrays of quantum dots containing a few ${ }^{4,11}$ or up to a few hundred electrons ${ }^{5}$ have confirmed the basic two-peak structure in the absorption (transmission) spectra. Small deviations were explained by taking into account the nonparabolicity effects, ${ }^{12}$ lateral Coulomb coupling between the neighboring dots, ${ }^{13}$ and spinorbit interaction. ${ }^{1}$ In a separate line of development, experimentalists performed a FIR spectroscopy study ${ }^{14}$ of the formation of incompressible edge stripes ${ }^{15}$ in quantum dots and antidots with a deliberately tailored hard-wall confinement, thereby demonstrating that FIR spectroscopy is capable of giving a detailed insight into many-body systems. As a matter of fact, in this work in order to increase the signal strength and create nonparabolic potential profiles, double-layer dots with three doping layers were used. Therefore, besides the dominant "optical" modes where the electrons in both layers oscillate in phase, also somewhat weaker "acoustic" modes were visible.

Recently, the system of two vertically coupled quantum dots was also studied theoretically in greater detail. ${ }^{16}$ This setup is interesting because even in the case when the confining potentials of the individual dots are parabolic, the Kohn theorem is broken by the interaction between the nonequivalent dots. Therefore, besides the usual c.m. modes a rather rich spectrum of collective modes can be excited, however, most of the oscillator strength is still contained in the center-of-mass modes.

In the present paper we study a similar system consisting of two vertically coupled circular parabolic quantum dots containing carriers of opposite charge sign, electrons and holes, respectively. Our results are very different from those pertaining to the previously studied ${ }^{16}$ vertically coupled dot system and reveal an interesting anticrossing of c.m.-derived modes belonging to separate dots marked by a major oscillator strength transfer between them. The kind of system we have in mind can be structured in bilayer-bipolar heterostructures containing parallel electron and hole layers in equilibrium. These structures have been realized in the crossed gap InAs/GaSb system ${ }^{17}$ as well as in biased GaAs/ $/ \mathrm{Al}_{x} \mathrm{Ga}_{1-x} \mathrm{As}$ heterostructure ${ }^{18,19}$ where electron and hole layers form on the opposite sides of an $\mathrm{Al}_{x} \mathrm{Ga}_{1-x} \mathrm{As}$ barrier. Most of the interest in such systems stems from the possibility (at least, in principle) of the formation of Bose-Einstein condensate of indirect excitons. ${ }^{20}$ While the formation of the superfluid state has not been demonstrated so far, a number of other interesting effects due to the electron-hole coupling have been predicted and/or observed. ${ }^{19,21}$

Our computational approach is based on the generalization to bilayer two-component systems of the formalism developed by Zaremba and his co-worker ${ }^{22}$ and successfully applied to a number of electronic systems. ${ }^{23,24}$ This approach is well suited to describe quantum dots with a large number of electrons whose dynamic response is dominated by collective excitations. ${ }^{24}$ Our paper has the following structure. In Sec. II we discuss the equilibrium charge-density distribution in artificial bipolar molecules. The formalism is given in Sec. III, and the results regarding the dynamic response of these systems are presented in Sec. IV. We summarize our results in Sec. V. Two Appendixes describe, respectively, the calculation of Coulomb integrals and a simplified model useful for obtaining quick estimates of the essential characteristics of the spectrum.

\section{EQUILIBRIUM DENSITIES}

We consider two vertically coupled quantum dots, one populated by electrons and the other by an equal number of 
holes. Both electrons and holes are strictly two dimensional and are laterally confined by parabolic potentials.

The equilibrium and dynamical properties of a manyelectron system close to the classical regime can be calculated from an approximate semiclassical total-energy functional $^{23,24}$ of the electron density $n_{e}$

$$
\begin{aligned}
E_{e}\left[n_{e}\right]= & T\left[n_{e}\right]+\frac{1}{2} \int d^{2} r \int d^{2} r^{\prime} \frac{n_{e}(\mathbf{r}) n_{e}\left(\mathbf{r}^{\prime}\right)}{\left|\mathbf{r}-\mathbf{r}^{\prime}\right|} \\
& +\int d^{2} r w_{e}(\mathbf{r}) n_{e}(\mathbf{r})+E_{\mathrm{xc}}\left[n_{e}(\mathbf{r})\right] .
\end{aligned}
$$

Besides the largest contributions of the direct Coulomb interaction energy and the energy in the external confining potential $w_{e}$, the functional (1) includes the quantummechanical kinetic and exchange-correlation energy corrections. Following previous authors, ${ }^{23,24}$ we choose to represent the kinetic energy by its lowest-order gradient (von Weizsäcker) expansion and approximate $E_{\mathrm{xc}}$ by the local Dirac exchange-only term. We work in the effective atomic units defined by setting $\hbar=m_{e}^{*}=e^{2} / \epsilon=1$. Here $m_{e}^{*}$ is the effective electron mass (the effective hole mass may be different) and $\epsilon$ is the dielectric constant of the medium. In these units, the kinetic energy and exchange functionals, respectively, are given by

$$
\begin{gathered}
T\left[n_{e}\right]=\frac{\pi}{2} \int d^{2} r n_{e}^{2}(\mathbf{r})+\frac{\lambda}{8} \int d^{2} r \frac{\left|\nabla n_{e}(\mathbf{r})\right|^{2}}{n_{e}(\mathbf{r})}, \\
E_{x}\left[n_{e}\right]=-\frac{4}{3} \sqrt{\frac{2}{\pi}} \int d^{2} r\left[n_{e}(\mathbf{r})\right]^{3 / 2},
\end{gathered}
$$

where $\lambda=0.25$ is the von Weizsäcker coefficient. ${ }^{24}$

For a two-component system (electrons and holes) the total energy consists of the energies of the two subsystems and a coupling term

$$
E\left[n_{e}, n_{h}\right]=E_{e}\left[n_{e}\right]+E_{h}\left[n_{h}\right]+E_{\text {coup }}\left[n_{e}, n_{h}\right] .
$$

The energy functional of the holes $E_{h}\left[h_{h}\right]$ is identical in form to that of electrons as given in Eqs. (1) and (2), however, since the holes may have a different effective mass the kinetic-energy term is scaled by the inverse of the ratio of hole-to-electron effective masses $\kappa=m_{h}^{*} / m_{e}^{*}$. In our calculations we use the characteristic value $\kappa=3$. (Using the bulk GaAs data, ${ }^{25}$ one obtains $\kappa=7.9$ and 1.2 for heavy and light holes, respectively.) The interlayer coupling is included at the mean-field level

$$
E_{\text {coup }}\left[n_{e}, n_{h}\right]=-\int d^{2} r_{e} \int d^{2} r_{h} \frac{n_{e}\left(\mathbf{r}_{e}\right) n_{h}\left(\mathbf{r}_{h}\right)}{\left|\mathbf{r}_{e}-\mathbf{r}_{h}-\mathbf{d}\right|},
$$

with $\mathbf{d}$ denoting the vertical separation between the layers.

The equilibrium densities are obtained from the two Euler equations,

$$
\begin{aligned}
& \frac{\delta}{\delta n_{e}} E\left[n_{e}, n_{h}\right]=\mu_{e}, \\
& \frac{\delta}{\delta n_{h}} E\left[n_{e}, n_{h}\right]=\mu_{h} .
\end{aligned}
$$

Following the usual procedure ${ }^{23,24}$ of evaluating the functional derivatives in Eq. (4) and expressing them in terms of the square root of the particle densities $\psi_{e(h)}(\mathbf{r})$ $=\left[n_{e(h)}(\mathbf{r})\right]^{1 / 2}$ we arrive at the equations determining the equilibrium charge density profiles

$$
\begin{aligned}
& {\left[-\frac{\lambda}{2} \nabla^{2}+u_{e}(\mathbf{r})-\mu_{e}\right] \psi_{e}(\mathbf{r})=0,} \\
& {\left[-\frac{\lambda}{2 \kappa} \nabla^{2}+u_{h}(\mathbf{r})-\mu_{h}\right] \psi_{h}(\mathbf{r})=0,}
\end{aligned}
$$

with the following expressions for the effective potentials:

$$
\begin{aligned}
u_{e}(\mathbf{r})= & w_{e}(\mathbf{r})+\pi \psi_{e}^{2}(\mathbf{r})-\sqrt{\frac{8}{\pi}} \psi_{e}(\mathbf{r}) \\
& +\int d^{2} r^{\prime} \frac{\psi_{e}^{2}\left(\mathbf{r}^{\prime}\right)}{\left|\mathbf{r}-\mathbf{r}^{\prime}\right|}-\int d^{2} r^{\prime} \frac{\psi_{h}^{2}\left(\mathbf{r}^{\prime}\right)}{\left|\mathbf{r}-\mathbf{r}^{\prime}-\mathbf{d}\right|}, \\
u_{h}(\mathbf{r})= & w_{h}(\mathbf{r})+\frac{1}{\kappa} \pi \psi_{h}^{2}(\mathbf{r})-\sqrt{\frac{8}{\pi}} \psi_{h}(\mathbf{r}) \\
& +\int d^{2} r^{\prime} \frac{\psi_{h}^{2}\left(\mathbf{r}^{\prime}\right)}{\left|\mathbf{r}-\mathbf{r}^{\prime}\right|}-\int d^{2} r^{\prime} \frac{\psi_{e}^{2}\left(\mathbf{r}^{\prime}\right)}{\left|\mathbf{r}-\mathbf{r}^{\prime}-\mathbf{d}\right|}
\end{aligned}
$$

Since the potentials (6) themselves depend on the solutions of Eq. (5), the system of equations (5) and (6) has to be solved self-consistently by convergent iterations. The angular integrations in Coulomb integrals appearing in Eqs. (5) and (6) can be carried out analytically as described in Appendix A. The remaining radial equations for $\psi_{e(h)}$ are solved numerically by discretizing the functions and potentials on a grid and using an imaginary-time evolution technique described in Ref. 24.

The results obtained using the above formalism are illustrated in Fig. 1. In our calculations we use GaAs material parameters $m_{e}^{*}=0.067 m_{e}$ and $\epsilon=13.4$ which define the effective Bohr radius (the length unit) $a_{B}^{*}=\hbar^{2} \epsilon / m_{e}^{*} e^{2}$

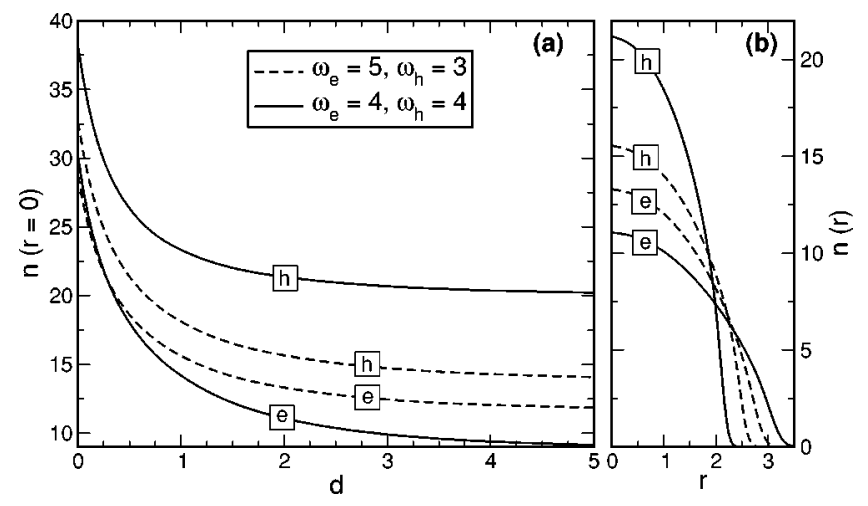

FIG. 1. Equilibrium particle density distributions in bipolar quantum-dot molecules. Panel (a) depicts the evolution of the particle densities at the center of the dots versus the interdot distance for equal (full line) and different (dashed line) confining frequencies. Panel (b) shows the radial dependences of the electron and hole densities for the two situations of (a) at $d=2$. 
$\approx 10 \mathrm{~nm}$ and the effective Hartree (the energy unit) $E_{H}^{*}$ $=e^{2} / a_{B}^{*} \approx 10 \mathrm{meV}$. Likewise, we express frequencies in the units $E_{H}^{*} / \hbar \approx 1.5 \times 10^{13} \mathrm{~s}^{-1}$, and the density unit is $a_{B}^{*-2}$ $=10^{16} \mathrm{~m}^{-2}$. In panel (b) of Fig. 1, we show two typical examples of the radial distribution of the particle densities in the dots obtained by setting the confining potentials to $\omega_{e}$ $=5, \omega_{h}=3$ (dashed line), and $\omega_{e}=\omega_{h}=4$ (full line), respectively. The interdot separation is $d=2$, and each dot contains an equal number $N_{e}=N_{h}=200$ of particles. We note that near the center the density profiles closely follow the usual semielliptic shape, ${ }^{8}$ while at the edges the densities are somewhat smoothed if compared to the abrupt square-root behavior predicted by the classical treatment. ${ }^{8,16}$ This effect is due to the included quantum-mechanical corrections that make the density to approach zero asymptotically in the classically forbidden region. ${ }^{22}$ Panel (a) displays the particle densities at the central axis of the quantum-dot molecule (i.e., the centers of the two dots) as a function of the interdot distance $d$. Here we use the same values of $N_{e}=N_{h}=200$ and two different sets of confining frequencies. The full lines are obtained by setting $\omega_{e}$ and $\omega_{h}$ to the same value 4 ; in this case, due to a higher hole effective mass the radius of the dot containing holes is smaller and the hole densities at the center are considerably larger if compared to the electronic dot. The dashed lines illustrate the case of a quantum-dot molecule with better balanced radii and densities of its two components. Here the confining frequencies are set to $\omega_{e}=5$ and $\omega_{h}=3$. We observe that in all cases the particle densities take off rapidly, and hence the dot radii shrink, when the inter-dot distance $d$ becomes comparable to or smaller than the dot radii which are typically in the range $2 \leq R \leq 3.5$.

\section{DYNAMIC RESPONSE-THEORY}

When the electron-hole system is perturbed away from the equilibrium there develops an internal restoring force. The scalar potentials of its components $\Phi_{e(h)}$ acting on electron and hole subsystems are given by the functional derivatives of the total-energy functional with respect to the component densities evaluated at the modified density values ${ }^{23,24}$

$$
\mathbf{F}_{e(h)}^{\mathrm{int}}=-\nabla \Phi_{e(h)} \quad \text { with } \Phi_{e(h)}=\frac{\delta}{\delta n_{e(h)}} E\left[n_{e}, n_{h}\right] .
$$

This internal force, along with the external force due to the electric field of the FIR radiation and the Lorentz force in the presence of a perpendicular magnetic field, enters the set of four linearized hydrodynamic equations: the continuity and the force-balance equations for each of the two components. However, in order to avoid repetition, we will write out explicitly and manipulate only two generic equations,

$$
\begin{gathered}
\frac{\partial}{\partial t} n^{1}+\boldsymbol{\nabla} \cdot\left[n^{0} \mathbf{v}\right]=0, \\
\kappa \frac{\partial}{\partial t} \mathbf{v}=-\nabla \Phi+\eta e \mathbf{E}+\eta \mathbf{v} \times \vec{\omega}_{c} .
\end{gathered}
$$

The equations describing the hole (electron) layer are obtained from Eq. (8) by setting the charge-sign factor $\eta=+1$ $(-1)$ and supplying an extra subscript $h(e)$ to the density, velocity and potential fields. In addition, for electrons one sets the effective-mass factor to $\kappa=1$. In Eq. (8), the Lorentz force is expressed in terms of the vector $\vec{\omega}_{c}$ directed perpendicular to the layers whose absolute value equals the cyclotron-resonance frequency of the electrons.

The quantities $n^{0}$ and $n^{1}$ denote, respectively, the equilibrium density and the linear-order deviation. We work with stationary fields that depend on time as $e^{-i \omega t}$ and consider only dipole excitations of a given circular polarization. Therefore, in our square root of density notation we express the densities as

$$
n=\left(\psi+\phi e^{i \theta}\right)^{2},
$$

here $\theta$ is the angular coordinate. Thus, the two ground-state densities equal $n_{e(h)}^{0}=\psi_{e(h)}^{2}$ and the first-order fluctuations are given by $n_{e(h)}^{1}=2 \psi_{e(h)} \phi_{e(h)} e^{i \theta_{e(h)}}$. Observe that the fields $\psi$ and $\phi$ are both circularly symmetric, and the correct angular dependence of $n^{1}$ is explicitly included in the factors $e^{i \theta}$. The external electric field is also taken to be circularly polarized and derivable from its corresponding scalar potential $\mathbf{E}=-\nabla \Phi_{\mathrm{ext}}$ with $\Phi_{\mathrm{ext}}=-E r e^{i \theta}$ and $E=$ const. We note that it is not necessary to consider also the opposite polarization $\sim e^{-i \theta}$ since these results can be obtained from the same calculation by simply changing the direction of $\vec{\omega}_{c}$, i.e., the sign of its vertical projection. Positive (negative) values of $\omega_{c}$ correspond to the direction of the electron (hole) cyclotron resonance. Substituting the equilibrium and oscillating densities from Eq. (9) into Eqs. (8) we obtain

$$
\begin{gathered}
-i \omega 2 \psi \phi e^{i \theta}+\operatorname{div}\left(\psi^{2} \mathbf{v}\right)=0, \\
-i \tilde{\omega} \kappa \mathbf{v}=-\nabla \Phi+\eta e \mathbf{E}+\eta \mathbf{v} \times \vec{\omega}_{c},
\end{gathered}
$$

here we also allow for the presence of a small damping force, thus making a replacement $\omega \rightarrow \tilde{\omega}=\omega-i \gamma$ in Eq. (10b).

The force-balance equation is readily solved by taking its cross product with $\vec{\omega}_{c}$ and using the result to eliminate the cross-product term in Eq. (10b). Straightforward algebra gives

$$
\begin{aligned}
\left(\omega_{c}^{2}-\kappa^{2} \tilde{\omega}^{2}\right) \mathbf{v}= & {\left[i \kappa \tilde{\omega} \nabla \Phi-\eta \nabla \Phi \times \vec{\omega}_{c}\right] } \\
& +\left[e \mathbf{E} \times \vec{\omega}_{c}-i \eta \kappa \tilde{\omega} e \mathbf{E}\right] .
\end{aligned}
$$

Substituting this expression into the continuity equation we will need only the divergence and the radial component of the velocity field. We separate out the angular dependence of $\Phi$ by writing it as $\Phi=f(r) e^{i \theta}$, and carrying out the derivative calculations we obtain the wanted quantities

$$
\left(\omega_{c}^{2}-\kappa^{2} \tilde{\omega}^{2}\right) v_{r}=e^{i \theta}\left(i \tilde{\omega} \kappa f^{\prime}-\eta \omega_{c} \frac{i}{r}-i \eta \tilde{\omega} \kappa e E+i \omega_{c} e E\right),
$$

and

$$
\left(\omega_{c}^{2}-\kappa^{2} \tilde{\omega}^{2}\right) \operatorname{div} \mathbf{v}=i \tilde{\omega} \kappa \nabla^{2} \Phi=i \tilde{\omega} \kappa e^{i \theta} \Delta_{r} f .
$$


Here the operator

$$
\Delta_{r}=\frac{d^{2}}{d r^{2}}+\frac{1}{r} \frac{d}{d r}-\frac{1}{r^{2}}
$$

denotes the radial part of the Laplacian.

Putting everything together we end up with the following generic equation for the charge density fluctuation $\phi$ :

$$
\begin{aligned}
& -\omega\left(\omega_{c}^{2}-\kappa^{2} \tilde{\omega}^{2}\right) \phi+\tilde{\omega} \kappa\left(f^{\prime} \psi^{\prime}+\frac{1}{2} \Delta_{r} f \psi\right)-\omega_{c} \eta f \frac{1}{r} \psi^{\prime} \\
& =e E \psi^{\prime}\left(\eta \tilde{\omega} \kappa-\omega_{c}\right)
\end{aligned}
$$

which will generate the two equations for the electron and hole layers. We stress that the quantities $f$ (radial parts of the potentials $\Phi)$ themselves are linear functionals of $\phi$ 's. Therefore, the right-hand side of Eq. (11) can be represented as a result of an application of a certain linear operator on $\phi$.

We write the internal potentials $\Phi_{e}$ and $\Phi_{h}$ as

$$
\Phi_{e}=\Phi_{e e}+\Phi_{e h} \text { and } \Phi_{h}=\Phi_{h e}+\Phi_{h h},
$$

thus separating the internal potentials created by interlayer and intralayer interactions. The expressions of the respective contributions are obtained by straightforward functional differentiation from the definition (7) and read

$$
\begin{aligned}
\Phi_{e e}= & 2 \pi \psi_{e} \phi_{e}-\frac{\lambda}{2} \psi_{e}^{-2}\left(\psi_{e} \nabla^{2} \phi_{e}-\phi_{e} \nabla^{2} \psi_{e}\right) \\
& -\sqrt{\frac{8}{\pi}} \phi_{e}+2 \int d^{2} r^{\prime} \frac{\psi_{e}\left(\mathbf{r}^{\prime}\right) \phi_{e}\left(\mathbf{r}^{\prime}\right)}{\left|\mathbf{r}-\mathbf{r}^{\prime}\right|} \\
\Phi_{h h}= & \frac{2 \pi}{\kappa} \psi_{h} \phi_{h}-\frac{\lambda}{2 \kappa} \psi_{h}^{-2}\left(\psi_{h} \nabla^{2} \phi_{h}-\phi_{h} \nabla^{2} \psi_{h}\right) \\
& -\sqrt{\frac{8}{\pi}} \phi_{h}+2 \int d^{2} r^{\prime} \frac{\psi_{h}\left(\mathbf{r}^{\prime}\right) \phi_{h}\left(\mathbf{r}^{\prime}\right)}{\left|\mathbf{r}-\mathbf{r}^{\prime}\right|} \\
& \Phi_{e h}=-2 \int d^{2} r^{\prime} \frac{\psi_{h}\left(\mathbf{r}^{\prime}\right) \phi_{h}\left(\mathbf{r}^{\prime}\right)}{\left|\mathbf{r}-\mathbf{r}^{\prime}-\mathbf{d}\right|} \\
& \Phi_{h e}=-2 \int d^{2} r^{\prime} \frac{\psi_{e}\left(\mathbf{r}^{\prime}\right) \phi_{e}\left(\mathbf{r}^{\prime}\right)}{\left|\mathbf{r}-\mathbf{r}^{\prime}-\mathbf{d}\right|} .
\end{aligned}
$$

The Coulomb integrals entering the expressions in Eqs. (12) resemble those encountered in the calculation of the equilibrium properties in Eqs. (5) and (6). However, in the present case we deal with $p$-wave charge distributions of angular dependence $\sim e^{i \theta}$ creating $p$-wave electrostatic potentials. The calculation of these integrals is also discussed in Appendix A.

For the sake of notational compactness, we introduce linear operators $\mathcal{L}$ corresponding to the different terms in these expressions, so that Eq. (11) can be written as

$$
\begin{gathered}
-\omega\left(\omega_{c}^{2}-\tilde{\omega}^{2}\right) \phi_{e}+\mathcal{L}^{e e} \phi_{e}+\mathcal{L}^{e h} \phi_{h}=R^{e}, \\
-\omega\left(\omega_{c}^{2}-\kappa^{2} \tilde{\omega}^{2}\right) \phi_{h}+\mathcal{L}^{h e} \phi_{e}+\mathcal{L}^{h h} \phi_{h}=R^{h},
\end{gathered}
$$

where $R^{e}=-e E \psi^{\prime}\left(\tilde{\omega}+\omega_{c}\right)$ and $R^{h}=e E \psi^{\prime}\left(\tilde{\omega} \kappa-\omega_{c}\right)$ stand for expressions on the right-hand side of Eq. (11).

We choose to expand the fields $\phi_{e} e^{i \theta_{e}}$ and $\phi_{h} e^{i \theta_{e}}$ in the set of Darwin-Fock functions of the angular momentum $M$ $=1$. Thus,

$$
\begin{gathered}
\phi_{e}=\frac{1}{r_{0}} \sum_{n=0}^{\infty} a_{n} g_{n}\left(r / r_{0}\right), \\
\phi_{h}=\frac{1}{r_{0}} \sum_{n=0}^{\infty} b_{n} g_{n}\left(r / r_{0}\right)
\end{gathered}
$$

with $a_{n}$ and $b_{n}$ being the expansion coefficients and the radial functions,

$$
g_{n}(r)=\sqrt{\frac{2}{n+1}} e^{-r^{2} / 2} r L_{n}^{1}\left(r^{2}\right)
$$

are expressed in terms of the associated Laguerre polynomials $L_{n}^{1}(x)$. The expansions (14) can be optimized by tuning the scaling radius $r_{0}$, and typically some 20 terms are needed in Eqs. (14) to obtain convergent results.

The remaining task is the numerical calculation of the matrix elements of the operators $\mathcal{L}$ in the basis (14) leading to the coupled set of linear equations

$$
\begin{gathered}
-\omega\left(\omega_{c}^{2}-\tilde{\omega}^{2}\right) a_{n}+\sum_{n^{\prime}}\left(\mathcal{L}_{n n^{\prime}}^{e e} a_{n^{\prime}}+\mathcal{L}_{n n^{\prime}}^{e h} b_{n^{\prime}}\right)=R_{n}^{e}, \\
-\omega\left(\omega_{c}^{2}-\kappa^{2} \tilde{\omega}^{2}\right) b_{n}+\sum_{n^{\prime}}\left(\mathcal{L}_{n n^{\prime}}^{h e} a_{n^{\prime}}+\mathcal{L}_{n n^{\prime}}^{h h} b_{n^{\prime}}\right)=R_{n}^{h},
\end{gathered}
$$

which we solve numerically by lower-upper decomposition ${ }^{26}$ and obtain the sets of expansion coefficients $a_{n}$ and $b_{n}$. This enables us to reconstruct the fluctuating charge-density profiles $\phi_{e(h)}$ in the two layers and evaluate the energy dissipation due to the Joule heating,

$$
P_{e(h)}(\omega)=\mp 2 \pi e E \omega \operatorname{Im}\left[\int_{0}^{\infty} d r r^{2} \psi_{e(h)} \phi_{e(h)}\right] .
$$

The sum $P=P_{e}+P_{h}$ determines the absorption rate.

\section{DYNAMIC RESPONSE-NUMERICAL RESULTS}

Turning to the description of the absorption spectra of coupled bipolar quantum dots, we begin by discussing the four most conspicuous modes that evolve from the c.m. modes of the two individual dots. Later, we proceed to describe the higher resonances and low-frequency edge modes whose oscillator strengths are inherently weak thus rendering them more difficult to observe experimentally.

The basic structure of the c.m.-mode spectrum as a function of magnetic field is shown in Fig. 2. These results are obtained for coupled dots containing $N_{e}=N_{h}=200$ particles each with confinement frequencies set to $\omega_{e}=5$ and $\omega_{h}=3$ (as in the second example of Sec. II). The vertical separation between the dots is $d=3$. The encircled symbols " + " and "- " designate the directions of the circular polarizations of 


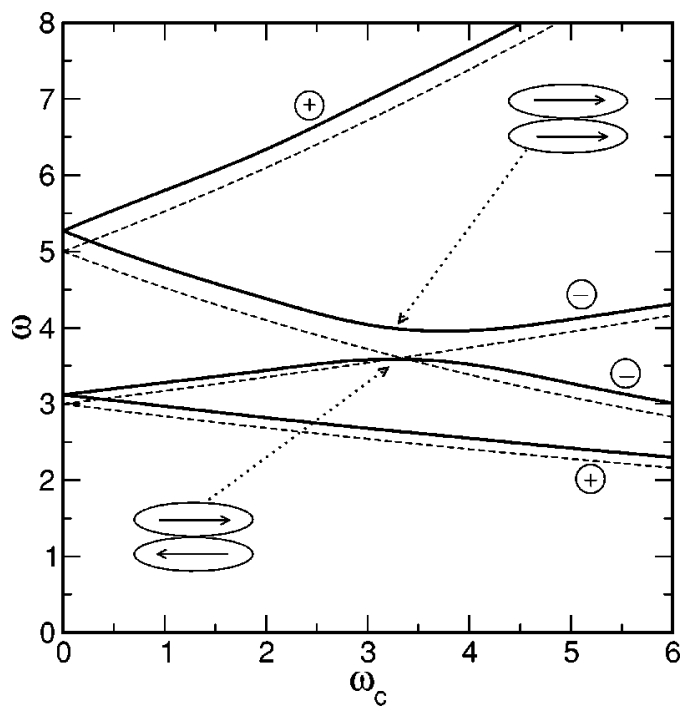

FIG. 2. The magnetic-field dependence of the four principal modes in the spectrum of a bipolar quantum-dot molecule. Symbols "+" and "-" indicate the polarizations. Full lines correspond to the vertical separation of $d=3$, and dashed lines depict the decoupled limit $d=\infty$. The insets illustrate the relative arrangements of electrical dipoles of the two dots pertinent to the respective anticrossing branches.

the respective modes. Our convention is to take the direction of the electronic cyclotron resonance as "positive" $(+)$ and vice versa. The narrow dashed lines indicate the positions of the $\mathrm{CM}$ modes of decoupled dots (i.e., $d=\infty$ ). In agreement with the generalized Kohn theorem, at zero magnetic field their frequencies coincide with the confinement frequencies $\omega_{e}$ and $\omega_{h}$, and split into two branches at finite magnetic fields. One notes that due to the Coulomb coupling between the dots, (i) all the modes are slightly displaced upwards with respect to their positions at $d=\infty$, and (ii) the two middle modes which are polarized in the same "-" direction anticross, while the two modes of " + " polarization reside in distinct frequency regions and thus interact only very weakly. Both points indicate significant differences from the spectra of vertically coupled electronic quantum dots, ${ }^{16}$ where the interdot coupling induces shifts of the modes towards lower frequencies and no such anticrossing is observed. In these systems, the interaction only couples pairs of c.m. modes that both have positive or negative magneticfield dispersion and do not cross in the absence of interaction. Thus we see that the charge-sign reversal of particles in one of the dots does indeed induce a substantial qualitative difference. In Appendix B, we show that essential features of the c.m. mode spectrum can be captured within a simplified coupled harmonic-oscillator model that can be useful in obtaining quick estimates.

We take a closer look at the anticrossing modes in Figs. 3 and 4 which show, respectively, the behavior of the frequencies of the two anticrossing branches and their oscillator strengths for three different values of $d$. We note from Fig. 3 that as the interdot separation becomes smaller and the coupling between the dots increases, the anticrossing becomes more pronounced while at the same time both branches tend

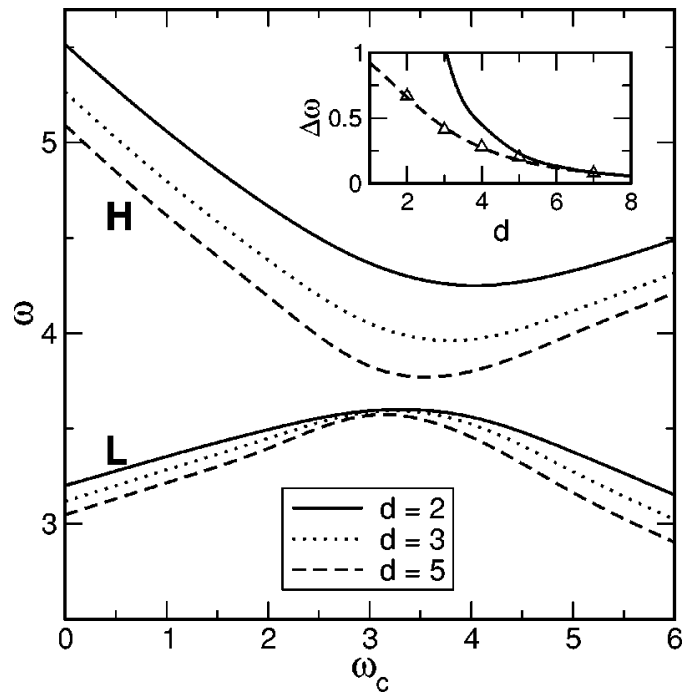

FIG. 3. The magnetic-field dispersion of the anticrossing c.m. modes of negative polarization plotted for three different values of the vertical interdot separation $d$. The symbols " $H$ " and " $L$ " label the higher and the lower anticrossing branches. Note the widening of the anticrossing gap and its shift towards higher frequencies with decreasing $d$. The inset compares the gap widths obtained from a numerical calculation (triangles) and its fit (dashed line) to those obtained from the simplified model of Appendix B (full line).

to shift towards higher frequencies. The inset of Fig. 3 shows the dependence of the size of the anticrossing gap $\Delta \omega$ on the interdot separation $d$. The triangles denote the actual calculated values while the full line shows the result obtained from a simple harmonic-oscillator model discussed in Ap-

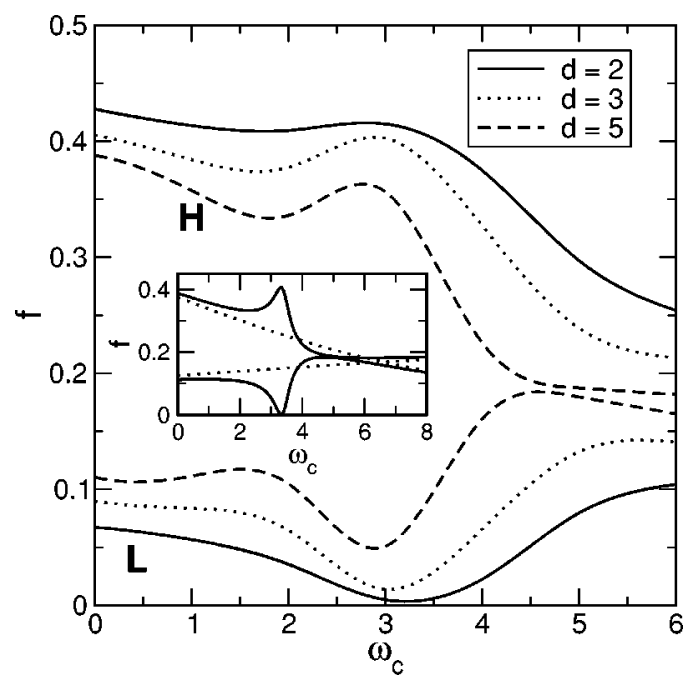

FIG. 4. The oscillator strengths of the two anticrossing c.m. modes of the negative polarization. The upper (lower) family of curves corresponds to the optical (acoustic) branch. A pronounced oscillator strength transfer between the modes in the magnetic-field range $2<\omega_{c}<4$ is apparent. As in Fig. 3, the symbols " $H$ " and " $L$ " indicate the higher and the lower anticrossing branches, respectively. The inset depicts the results obtained from the simplified model described in Appendix B. The full (dotted) lines correspond to coupled (uncoupled) dots. 
pendix B. This model predicts that the gap grows proportionally to $\Delta \omega \sim d^{-3}$, however, this law is valid only at relatively large distances $d>5$. We found that for smaller values of $d$ the splitting could be reasonably well fitted by the dependence $\Delta \omega \sim\left(d^{2}+d_{0}^{2}\right)^{-3 / 2}$ indicated by a dashed line in the inset of Fig. 3.

The evolution of the respective oscillator strengths in Fig. 4 is rather peculiar and requires a more detailed explanation. The sum of the oscillator strengths satisfies a sum rule, and we normalize it so that their total sum equals 1 . In the present example the electron and hole numbers are set equal while the holes are taken to be $\kappa=3$ times heavier than the electrons. Therefore, since the oscillator strengths scale as $\sim N / m$, the electronic modes possess three times higher oscillator strengths. Thus, at zero magnetic field the oscillator strengths of the two anticrossing modes start from the values close to 0.125 for the lower-energy mode that is essentially localized in the hole subsystem and 0.375 for the higher one. These numbers are slightly modified due to the interaction between the modes (the role of interaction becomes more important at lower values of $d$ ) as well as due to the presence of other much weaker modes. The rest $50 \%$ of the total oscillator strength at $\omega_{c}=0$ belongs to the modes of the opposite "+" polarization not shown here.

In the range of magnetic-field strengths $2<\omega_{c}<4$ the two modes interact strongly and anticross. In this region, one observes a rather pronounced depression in the oscillator strength of the lower branch reaching nearly zero value. The missing oscillator strength is transferred to the highfrequency branch. This type of behavior can be understood by realizing that at the anticrossing point the charge-density oscillations of the individual dots combine together in either "optical" (the two electric dipoles being aligned in parallel) or "acoustic" (antiparallel dipoles) manner, as illustrated in the insets of Fig. 2. Naturally, the parallel alignment of two dipoles costs more energy, and therefore this optical mode has a higher oscillation frequency, while at the same time it possesses a larger net dipole moment, and consequently, a higher oscillator strength. As one notes in the inset of Fig. 4, the same type of qualitative behavior is also observed in the coupled harmonic-oscillator model of Appendix B. However, as far as oscillator strengths are concerned, its quantitative predictions are not trustworthy, and thus one can only rely on the more-accurate numerical treatment. Our calculations predict the above described oscillations of the oscillator strengths to be quite strong. In Fig. 5 we show a set of absorption lines simulating those obtainable in FIR spectroscopy measurements ${ }^{1,6,14}$ which have been calculated for the case of a vertical separation between the dots $d=3$. We plot nine lines corresponding to nine equally spaced values of the magnetic fields between $\omega_{c}=1$ and $\omega_{c}=5$ (the anticrossing region) thus making the "disappearance" of the lowfrequency branch apparent. Since the fluctuations of the oscillator strengths of these modes can be rather large (of order of $10 \%$ of the total oscillator strength) we expect that the above described effect could be readily observed experimentally. It is worth mentioning that while in the above examples we always dealt with equal numbers of electrons and holes in the dots, the ratio of the electron and hole numbers can be

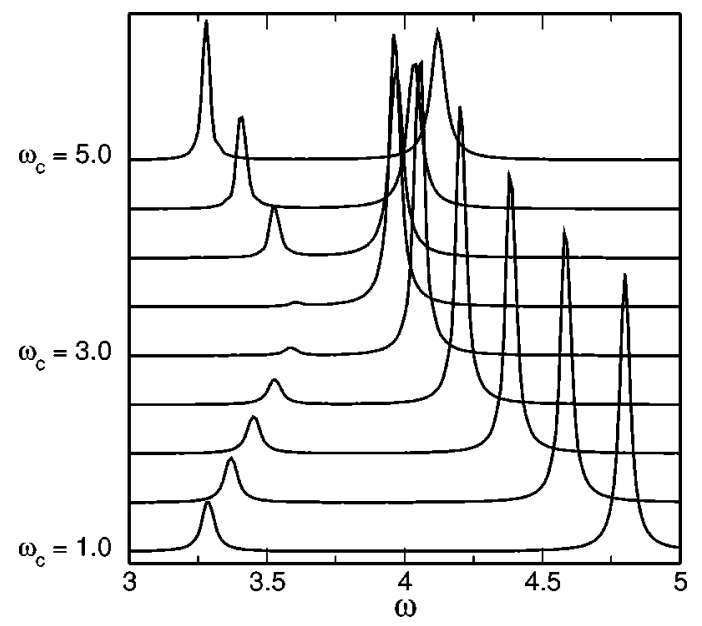

FIG. 5. FIR absorption (in arbitrary units) in a bipolar quantumdot molecule close to the anticrossing region. We show nine absorption curves corresponding to evenly spaced magnetic-field values and offset vertically by the same amount for clarity. Note the "disappearance" of the lower branch. A broadening $\gamma=0.05$ has been used.

useful to balance the distribution of oscillator strengths between the modes. For example, increasing the number of holes will brighten the modes that are mostly due to oscillations in the hole subsystem thereby compensating for their diminished oscillator strengths because of higher hole effective mass. As we show in Appendix B, the strength of the interaction between the two dots and the anticrossing gap scale as the geometric mean of the electron and hole numbers.

Besides the above described strong modes the spectra of artificial molecules feature a number of other rather weak modes that can be classified into higher resonances and lowenergy edge modes. Due to a stronger localization of the charge-density oscillations associated with these modes, the number of terms in the expansions (14) has to be increased for an accurate representation. The oscillator strengths of these modes, under the conditions of our calculations, typically barely reach $10^{-4}$ of the total sum of the oscillator strengths. In Fig. 6, we show the magnetic-field dispersions of the lowest-lying high-energy modes and several strongest edge modes. These results are obtained for the parameter values $N_{e}=N_{h}=200, \omega_{e}=4, \omega_{h}=3$, and $d=5$. The full (dashed) lines denote branches polarized in the positive (negative) direction. In general, the locations and dispersions of these modes resemble analogous modes as predicted for coupled quantum dots populated solely by electrons. ${ }^{16}$ The key difference is that in the present case one can classify the modes into those dominated by oscillations in either the electron or hole subsystems. Thus, the two lower high-energy resonances in Fig. 6 are mostly due to holes while the topmost mode is mainly electronic. The difference can be most easily spotted in the relative arrangement of the positively and negatively polarized branches at finite magnetic fields. In the electronic mode, the upper branch is polarized in the positive (i.e., electronic cyclotron resonance) direction, while in the case of the other two high-energy modes the situation 


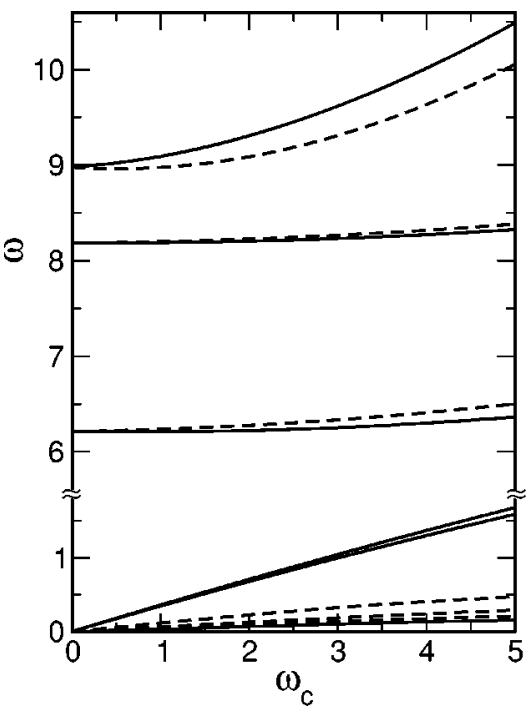

FIG. 6. The magnetic-field dispersion of the higher resonances and edge modes in a bipolar quantum-dot molecule. Note that the middle part of the spectrum is cut out. The full and dashed lines denote the modes of the positive and negative polarizations, respectively.

is reversed. Moreover, the electronic mode features a considerably stronger magnetic-field dispersion. This is due to the fact that the higher resonances asymptotically approach the cyclotron-resonance line, which is $\kappa=3$ times steeper for electrons than for holes.

The lower part of Fig. 6 shows six most conspicuous edge modes of the bipolar quantum-dot molecule. The frequency range covered by these modes and their dispersion again resemble the case of electronic coupled dots. ${ }^{16}$ However, due to the fact that the polarizations of the edge modes are determined by the charge sign of the carriers, in the present case the edge modes can be polarized both in the cyclotron and the anticyclotron directions. Moreover, the direction of polarization of an edge mode also betrays the component whose contribution is dominant. Thus, the edge modes mostly influenced by the electronic subsystem are polarized in the "-," i.e., anticyclotron direction of the electrons. These modes are depicted by the dashed lines in Fig. 6. On the contrary, the full lines in Fig. 6 denote the modes mostly due to the oscillations in the hole subsystem which are polarized in the "+," i.e., electronic cyclotron-resonance direction.

\section{SUMMARY}

In conclusion, we made a theoretical investigation of the equilibrium density distributions and the far-infrared response of bipolar quantum-dot molecules within a hydrodynamic model including the effects due to exchange and kinetic energy in the von Weizsäcker approximation. The most conspicuous effect we found is the pronounced anticrossing between two modified center-of-mass modes, which takes place when the applied magnetic field aligns the frequencies of two center-of-mass modes. The additional distinguishing feature of this anticrossing is the strongly nonmonotonous behavior of the oscillator strengths of the two resulting branches. The oscillator strength transfer from the lower "acoustic" to the higher "optical" branch is certainly strong enough to be easily observable. On the other hand, the higher resonances and edge modes which are also excited in the considered setup, are quite weak.

\section{ACKNOWLEDGMENTS}

This work was supported by the Inter-University Attraction Poles (IUAP-V) program, concerted action (GOA) and the University of Antwerp through a VIS project. E.A. was partially supported by EU under Contract No. HPMF-CT2001-01195. The authors acknowledge enlightening discussions with A. Matulis.

\section{APPENDIX A: COULOMB INTEGRALS}

In this paper we encountered two types of Coulomb integrals

$$
\begin{gathered}
J_{s}(r)=\int d^{2} r^{\prime} \frac{\psi_{0}^{2}\left(r^{\prime}\right)}{\left|\mathbf{r}-\mathbf{r}^{\prime}-\mathbf{d}\right|}, \\
J_{p}(\mathbf{r})=2 \int d^{2} r^{\prime} \frac{\psi_{0}\left(r^{\prime}\right) \psi_{1}\left(\mathbf{r}^{\prime}\right)}{\left|\mathbf{r}-\mathbf{r}^{\prime}-\mathbf{d}\right|} .
\end{gathered}
$$

Here, $\psi_{0}(r)$ depends only on the radial coordinate $r$ while $\psi_{1}(\mathbf{r})=f_{1}(r) e^{i \theta}$ has a $p$-wave angular dependence. $d$ is the vertical separation between the layers that as a special case may equal zero.

The angular integration in Eq. (A1) can be carried out analytically in terms of the complete elliptic functions of the first kind $K(k)$, and the second kind $E(k)$. Introducing $\rho^{2}$ $=\left(r+r^{\prime}\right)^{2}+d^{2}$ we obtain

$$
\begin{gathered}
J_{s}(r)=4 \int_{0}^{\infty} d r^{\prime} r^{\prime} \frac{\psi_{0}^{2}\left(r^{\prime}\right)}{\rho} K\left(\frac{\sqrt{4 r r^{\prime}}}{\rho}\right), \\
J_{p}(\mathbf{r})=8 e^{i \theta} \int_{0}^{\infty} d r^{\prime} r^{\prime} \frac{\psi_{0}\left(r^{\prime}\right) f_{1}\left(r^{\prime}\right)}{\rho} C\left(\frac{\sqrt{4 r r^{\prime}}}{\rho}\right),
\end{gathered}
$$

with

$$
C(k)=\frac{2}{k^{2}}[E(k)-K(k)]-K(k) .
$$

\section{APPENDIX B: COUPLED HARMONIC-OSCILLATOR MODEL}

Guided by a similar simple model introduced in Ref. 16, we show that a number of basic features of the c.m. mode spectrum can be derived (at least qualitatively) from a simplified model featuring two coupled harmonic oscillators. The oscillators concentrate the total masses and charges of the two coupled dots and interact via the potential 


$$
-\frac{N_{e} N_{h}}{\sqrt{d^{2}+\left(\mathbf{r}_{e}-\mathbf{r}_{h}\right)^{2}}} \approx-\frac{N_{e} N_{h}}{d}+\frac{N_{e} N_{h}}{2 d^{3}}\left(\mathbf{r}_{e}-\mathbf{r}_{h}\right)^{2} .
$$

Here $\mathbf{r}_{e}$ and $\mathbf{r}_{h}$ are the oscillator coordinates, and we used the fact that in our units $e^{2} / \epsilon=1$. Denoting the base frequencies of the oscillators by $\omega_{e}$ and $\omega_{h}$ we write down the coupled equations of motion $\left(m_{e}^{*}=1, m_{h}^{*}=\kappa\right)$

$$
\begin{array}{r}
N_{e} \ddot{\mathbf{r}}_{e}+N_{e} \omega_{e}^{2} \mathbf{r}_{e}+\frac{N_{e} e}{c} \dot{\mathbf{r}}_{e} \times \mathbf{B}+\frac{N_{e} N_{h}}{d^{3}}\left(\mathbf{r}_{e}-\mathbf{r}_{h}\right)=0, \\
N_{h} \kappa \ddot{\mathbf{r}}_{h}+N_{h} \kappa \omega_{h}^{2} \mathbf{r}_{h}-\frac{N_{h} e}{c} \dot{\mathbf{r}}_{h} \times \mathbf{B}-\frac{N_{e} N_{h}}{d^{3}}\left(\mathbf{r}_{e}-\mathbf{r}_{h}\right)=0 .
\end{array}
$$

Equations (B2) are solved by introducing the complex variables $z_{e(h)}=x_{e(h)}+i y_{e(h)}$ and assuming a harmonic temporal dependence $z_{e(h)} \sim \exp (i \omega t)$. This leads to the following secular equation for the resonance frequencies:

$$
\left|\begin{array}{ll}
-\omega^{2}+\omega_{e}^{2}+\omega \omega_{c}+N_{h} \Omega_{0}^{2} & -N_{h} \Omega_{0}^{2} \\
-N_{e} \Omega_{0}^{2} / \kappa & -\omega^{2}+\omega_{h}^{2}-\omega \omega_{c} / \kappa+N_{e} \Omega_{0}^{2} / \kappa
\end{array}\right|=0,
$$

here we denoted $\omega_{c}=e B / c$ and $\Omega_{0}^{2}=d^{-3}$. From Eq. (B3) the c.m. mode frequencies can be readily obtained as solutions of a quartic equation. However, basing on the smallness of the coupling parameter $\Omega_{0}^{2}$, it is possible to extract some simpler approximate expressions. We begin by noting that the role of the terms proportional to $\Omega_{0}^{2}$ entering the diagonal and off-diagonal matrix elements in Eq. (B3) is different. The former give first-order corrections to the frequency dispersion, while the latter contribute to the second order and are important only close to coinciding frequencies, thus defining an anticrossing.

Therefore, we first neglect the off-diagonal perturbations and solve two decoupled quadratic equations originating from the diagonal terms in Eq. (B3). The solutions read

$$
\begin{gathered}
\omega_{1,2}=\frac{\omega_{c}}{2} \pm \sqrt{\frac{\omega_{c}^{2}}{4}+\omega_{e}^{2}+N_{h} \Omega_{0}^{2}}, \\
\omega_{3,4}=-\frac{\omega_{c}}{2 \kappa} \pm \sqrt{\frac{\omega_{c}^{2}}{4 \kappa^{2}}+\omega_{h}^{2}+\frac{1}{\kappa} N_{e} \Omega_{0}^{2} .}
\end{gathered}
$$

The modes of positive frequencies $\omega_{1}$ and $\omega_{3}$ obtained using the upper signs in Eq. (B4) are of "+" circular polarization while the lower-sign solutions $\omega_{2}$ and $\omega_{4}$ are negative and correspond to modes polarized in the "-" direction. We note, that Eq. (B4) predicts that the absolute values of all four resonance frequencies increase due to the interdot cou-

*Electronic address: egidijus@uia.ua.ac.be

†Electronic address: peeters@uia.ua.ac.be

${ }^{1}$ L. Jacak, P. Hawrylak, and A. Wójs, Quantum Dots (Springer, Berlin, 1998)

${ }^{2}$ N. F. Johnson, J. Phys.: Condens. Matter 7, 965 (1995).

${ }^{3}$ L. P. Kouwenhoven, D. G. Austing, and S. Tarucha, Rep. Prog. pling and gives an estimate of the shifts. In the weakcoupling regime they grow as $\Omega_{0}^{2} \sim d^{-3}$. This conclusion is in agreement with our results (see Fig. 2) and underscores a difference of our system from electronic double dots considered in Ref. 16. There, all frequency shifts found were negative.

Assuming that (as in our numerical calculations) $\omega_{e}$ $>\omega_{h}$ we find that the modes polarized in "-" direction will cross. The crossing point is readily calculated by equating $\omega_{2}=\omega_{4}$ and equals

$$
\omega_{c 0}=\frac{\omega_{e}^{2}-\omega_{h}^{2}}{\sqrt{(1+1 / \kappa)\left(\omega_{e}^{2} / \kappa+\omega_{h}^{2}\right)}} .
$$

Note that we present the zero-order solution obtained by setting $\Omega_{0}^{2} \rightarrow 0$, which provides an accurate enough estimate. Close to this point the off-diagonal perturbations are important and have to be taken into account to introduce an anticrossing behavior. We use the fact that in the vicinity of the resonances of "-" polarization (roots $\omega_{2}$ and $\omega_{4}$ ) the diagonal terms of Eq. (B3) can be approximated by

$$
\begin{aligned}
& -\left(\omega-\omega_{1}\right)\left(\omega-\omega_{2}\right) \approx-\left(\omega_{2}-\omega_{1}\right)\left(\omega-\omega_{2}\right), \\
& -\left(\omega-\omega_{3}\right)\left(\omega-\omega_{4}\right) \approx-\left(\omega_{4}-\omega_{3}\right)\left(\omega-\omega_{4}\right),
\end{aligned}
$$

and obtain a quadratic equation

$$
\left(\omega-\omega_{2}\right)\left(\omega-\omega_{4}\right)\left(\omega_{1}-\omega_{2}\right)\left(\omega_{3}-\omega_{4}\right)-\frac{N_{e} N_{h}}{\kappa} \Omega_{0}^{4}=0
$$

valid in this region and giving an approximate behavior of the anticrossing modes. This equation can be used, in particular, to estimate the size of the anticrossing gap. To this end we calculate the difference of its two roots at $\omega_{c}=\omega_{c 0}$ and $\omega_{2}=\omega_{4}$, and obtain

$$
\Delta \omega=2 \Omega_{0}^{2} \sqrt{\frac{N_{e} N_{h}}{\kappa\left(\omega_{1}-\omega_{2}\right)\left(\omega_{3}-\omega_{4}\right)}},
$$

with $\omega_{1,2,3,4}$ given by Eq. (B4).

Equations (B4)-(B6) are useful as quick estimates of essential features (mode shifts, position and size of anticrossing gap) in the c.m. mode spectrum of bipolar quantum-dot molecules. These estimates are obtained for weakly coupled dots and can be asked for quantitave predictions only in the limit when interdot separation considerably exceeds the dot radii. Thus, the gap size obtained from Eq. (B6) agrees with the result of accurate numerical calculations within $10 \%$ at $d=5$. However, as we can see in the inset of Fig. 3 at closer distances the gap grows much more slowly than $\sim d^{-3}$ as given by Eq. (B6).
Phys. 64, 701 (2001).

${ }^{4}$ Ch. Sikorski and U. Merkt, Phys. Rev. Lett. 62, 2164 (1989).

${ }^{5}$ T. Demel, D. Heitmann, P. Grambow, and K. Ploog, Phys. Rev. Lett. 64, 788 (1990).

${ }^{6}$ D. Heitmann and J. P. Kotthaus, Phys. Today 46 (6), 56 (1993).

${ }^{7}$ D. Heitmann, Physica B 212, 201 (1995). 
${ }^{8}$ V. Shikin, S. Nazin, D. Heitmann, and T. Demel, Phys. Rev. B 43, 11903 (1991).

${ }^{9}$ A. Kumar, S. E. Laux, and F. Stern, Phys. Rev. B 42, 5166 (1990).

${ }^{10}$ L. Brey, N. F. Johnson, and B. I. Halperin, Phys. Rev. B 40, 10 647 (1989); P. A. Maksym and T. Chakraborty, Phys. Rev. Lett. 65, 108 (1990); F. M. Peeters, Phys. Rev. B 42, 1486 (1990); W. Kohn, Phys. Rev. 123, 1242 (1961).

${ }^{11}$ B. Meurer, D. Heitmann, and K. Ploog, Phys. Rev. Lett. 68, 1371 (1992)

${ }^{12}$ V. Gudmundsson and R. R. Gerhardts, Phys. Rev. B 43, 12098 (1991).

${ }^{13}$ T. Chakraborty, V. Halonen, and P. Pietiläinen, Phys. Rev. B 43, 14289 (1991).

${ }^{14}$ K. Bollweg, T. Kurth, D. Heitmann, V. Gudmundsson, E. Vasiliadou, P. Grambow, and K. Eberl, Phys. Rev. Lett. 76, 2774 (1996).

${ }^{15}$ C. W. J. Beenakker, Phys. Rev. Lett. 64, 216 (1990); D. B. Chklovskii, B. I. Shklovskii, and L. I. Glazman, Phys. Rev. B 46, 4026 (1992).

${ }^{16}$ B. Partoens, A. Matulis, and F. M. Peeters, Phys. Rev. B 57, 13 039 (1998).

${ }^{17}$ T. P. Marlow, L. J. Cooper, D. D. Arnone, N. K. Patel, D. M.
Whittaker, E. H. Linfield, D. A. Ritchie, and M. Pepper, Phys. Rev. Lett. 82, 2362 (1999); J. Kono, B. D. McCombe, J.-P. Cheng, I. Lo, W. C. Mitchel, and C. E. Stutz, Phys. Rev. B 55, 1617 (1997); M. Altarelli, ibid. 28, 842 (1983), and references therein.

${ }^{18}$ Yu. E. Lozovik and V. I. Yudson, Pis'ma Zh. Éksp. Teor. Fiz. 22, 556 (1975) [JETP Lett. 22, 274 (1975)].

${ }^{19}$ U. Sivan, P. M. Solomon, and H. Shtrikman, Phys. Rev. Lett. 68, 1196 (1992).

${ }^{20}$ Yu. E. Lozovik, O. L. Berman, and V. G. Tsvetus, Phys. Rev. B 59, 5627 (1999).

${ }^{21}$ Y. Naveh and B. Laikhtman, Europhys. Lett. 55, 545 (2001).

${ }^{22}$ E. Zaremba and H. C. Tso, Phys. Rev. B 49, 8147 (1994).

${ }^{23}$ See, for example, E. Zaremba, Phys. Rev. B 53, 10512 (1996); B. P. van Zyl and E. Zaremba, ibid. 59, 2079 (1999).

${ }^{24}$ B. P. van Zyl, E. Zaremba, and D. A. W. Hutchinson, Phys. Rev. B 61, 2107 (2000).

${ }^{25}$ P. Y. Yu and M. Cardona, Fundamentals of Semiconductors (Springer, Berlin, 2001), Chap. 2.

${ }^{26}$ W. H. Press, S. A. Teukolsky, W. T. Vetterling, and B. P. Flannery, Numerical Recipes in C (Cambridge University Press, Cambridge, 1994), Chap. 2. 